\title{
Expression of Hepatitis B Virus Surface Antigen (HBsAg) from Genotypes A, D and F and Influence of Amino Acid Variations Related or Not to Genotypes on HBsAg Detection
}

\author{
Natalia M. Araujo ${ }^{1}$, Carlos O. A. Vianna ${ }^{2}$, Marcia T. B. Moraes ${ }^{3}$ and Selma A. Gomes ${ }^{1}$ \\ ${ }^{1}$ Laboratory of Molecular Virology, Oswaldo Cruz Institute, FIOCRUZ; ${ }^{2}$ Vice Direction of Technological Development, Bio-Manguinhos, \\ FIOCRUZ; ${ }^{3}$ Laboratory of Recombinant Technology, Bio-Manguinhos, FIOCRUZ; Rio de Janeiro, RJ, Brazil
}

\begin{abstract}
The impact of hepatitis B virus (HBV) genotypes on the sensitivity of surface antigen (HBsAg) detection assays has been poorly investigated. Here, plasmids carrying consensus or variant coding sequences for $\mathrm{HBV}$ surface proteins from genotypes A, D and F, were constructed. HBsAg levels were evaluated in medium and extracts of transfected CHO cells by a commercial polyclonal-based assay. We show that HBsAg detection values of consensus forms from genotypes $\mathrm{D}$ and $\mathrm{F}$ were, respectively, $37 \%$ and $30 \%$ lower than those obtained by genotype $\mathrm{A}$. However, the presence of two single variations, T143M in genotype A, and T125M in genotype D, produced a decrease of $44 \%$ and an increase of $34 \%$, respectively, on HBsAg mean values in comparison with their consensus forms. In conclusion, HBsAg detection levels varied among HBV genotypes. However, unique amino acid substitutions not linked to genotypes, such as T125M and T143M described here, should have more implications in HBV immunological diagnostics than the set of variations characteristic of each HBV genotype.

Key-Words: Hepatitis B virus, surface antigen, amino acid variations, transfection.
\end{abstract}

Hepatitis B virus (HBV), the prototypic member of the family Hepadnaviridae, is an enveloped hepatotropic virus that causes acute and chronic human liver diseases, including cirrhosis and hepatocellular carcinoma. HBV has been classified into eight genotypes, designated $\mathrm{A}$ to $\mathrm{H}$, based upon genetic divergence of $8 \%$ or more in the complete nucleotide sequence [1-3]. HBV genotypes have a distinct prevalence in different geographical regions [4, 5]. In Brazil, a country whose population is miscigenated, HBV strains from genotypes A, D, and F are the most prevalent [6-8]. Genotype F, indigenous to South and Central Americas, is the most divergent genotype. Due to their restricted geographical area of prevalence, $\mathrm{HBV}$ isolates from genotype $\mathrm{F}$ have not been extensively studied with regard to immunological characteristics.

HBV surface antigen (HBsAg) consists of three structurally related envelope proteins: 1) small (S) protein (226 amino acid residues), the major constituent of HBV envelope protein; 2) middle (M) protein, containing additional 55 amino acid residues at the $\mathrm{N}$-terminus of S protein; 3) large (L) protein, containing a further 108 or 119 residues (according to genotype) at the N-terminus of M protein. All three forms, encoded by a unique open reading frame on the HBV genome, are cotranslationally inserted into the endoplasmic reticulum (ER) as transmembrane proteins. Virions and the abundant form of non-infectious $22 \mathrm{~nm}$-particles found in infected sera possess high quantities of S protein and lower quantities of $\mathrm{M}$ and L surface proteins [9-11].

Received on 28 March 2009; revised 15 July 2009.

Address for correspondence: Dr. Natalia M. Araujo. Laboratório de Virologia Molecular, Instituto Oswaldo Cruz, FIOCRUZ, Av. Brasil 4365, 21045-900 Rio de Janeiro, RJ, Brazil. Phone: +55 212562 1847; Fax: +55 212260 4866. E-mail address: nmaraujo@ioc.fiocruz.br.

The Brazilian Journal of Infectious Diseases

2009;13(4):266-271. (C) 2009 by The Brazilian Journal of Infectious Diseases and Contexto Publishing. All rights reserved.
The HBsAg main antigenic determinant ( $a$ determinant) is encoded by amino acid residues from 124 to 147 within the major hydrophilic loop of HBsAg (amino acids 101 to 164) [12]. The $a$ determinant is the major immune target for antibodies either used for immuno-prophylaxis or in assays for detection of HBsAg [13]. It has been demonstrated that some specific mutations in and around the $a$ determinant may change HBsAg antigenicity leading to escape detection by certain commercial HBsAg assays [14, 15]. The most relevant mutations seem to be the G145R, K141E and T131I, since they markedly affect the antigenic structure of HBsAg [16]. On the other hand, some natural amino acid variations in and around the $a$ determinant are linked to genotypes, as substitutions in non-conserved residues from positions 110, 114, 118, 122, 127, $131,134,140,143,158,159$ and $161[12,17]$. The impact of HBV genotypes on HBsAg detection has so far been poorly investigated. It has been related that HBV genotypes may influence the sensitivity of some HBsAg commercial assays [18].

Herein, recombinant plasmids coding consensus sequences for $\mathrm{M}$ protein from genotypes $\mathrm{A}, \mathrm{D}$ and $\mathrm{F}$ were constructed. Additionally, $\mathrm{M}$ and S plasmids carrying natural point variations within the $a$ determinant were also constructed. We investigated whether or not the genetic heterogeneity of these genotypes, may influence HBsAg detection by a commercial polyclonal-based assay. We also analyzed the influence of the different forms of HBV surface proteins on the patterns of HBsAg detection.

\section{Materials and Methods}

Construction of HBsAg Expression Plasmids

HBV DNA was extracted from $250 \mu \mathrm{L}$ of serum using phenol/chloroform [19]. PreS2/S and S genomic regions were PCR amplified in two independent assays. Sense oligonucleotide primers PS4H (5'CCCAAGCTTACACTCATCCTCAGGCCAT GCAGTG-3', nt positions 3194-3218) and S1H (5'- 
CCCAAGCTTCTTCTCGAGGACT GGGGACC-3', nt positions 124-143) were designed with a HindIII restriction site at their 5' end and used to amplify preS2/S and S regions, respectively. Two reverse primers, located at the same position (nt 819-841) in the HBV genome and designed with an EcoRV restriction site at their 5' ends, were utilized: S2E (5'- ATGGATATCG GGTTTAAATGTATACCCAAAGA-3') for amplification of HBV DNA from genotypes A and D, and S22E (5'ATGGATATCGTA TTTAAATGGATACCCACAGA-3’) for genotype F. PCR products were digested with HindIII and EcoRV and cloned into the polylinker region of the mammalian cell expression vector pcDNA3 (Invitrogen, San Diego, CA).

\section{Nucleotide Sequencing of Cloned HBV DNA}

Recombinant plasmids were purified by using S.N.A.P. Midiprep kit (Invitrogen). HBV nucleotide sequences were determined using BigDye Terminator kit (Applied Biosystems, Foster City, CA) with T7 and SP6 primers, flanking the vector cloning site, as well as specific internal HBV primers. Sequencing reactions were analyzed on ABI3730 automated sequencer (Applied Biosystems). Bioinformatics analysis of the sequences was performed using MEGA version 3.1 and BioEdit version 7.0.9.0.

\section{Transient Transfection Assays}

Transient transfection assays were performed using Chinese hamster ovary (CHO) cells grown in 199 Earle's medium supplemented with $10 \%$ fetal bovine serum. Cells were plated at a density of $5 \times 10^{5}$ cells per well of 6-well plates and transfected with $2 \mu \mathrm{g}$ of HBsAg expressing plasmid using FuGENE 6 (Roche Diagnostics, Mannheim, Germany), according to the manufacturer's instructions. Culture supernatants were collected on day 5 after transfection and clarified by centrifugation at 1,500g for $5 \mathrm{~min}$. To evaluate HBsAg levels inside cells, cell monolayers were washed twice with phosphate buffered saline (PBS), scraped with $1 \mathrm{~mL}$ of PBS and centrifuged at 2,500g for $5 \mathrm{~min}$. Cell pellets were freezed/thawed three times and resuspended in $0.4 \mathrm{~mL}$ of PBS. Medium and cell extracts were tested for the presence of HBsAg by a commercial immunoassay that uses polyclonal antibodies for both capture and detection (BioELISA HBsAg colour, Biokit, Barcelona, Spain). Mock-transfected CHO cells were used as negative control.

\section{Results}

Amino Acid Sequence Variations of the HBV Expression Plasmids

HBV DNA was extracted from a number of HBsAg positive serum samples and preS2/S region was amplified and sequenced. The genotypes of the HBV isolates were determined by the construction of a phylogenetic tree, comparing the PCR sequences to DNA database sequences from genotypes A, D and F (not shown). Three sera that showed HBV DNAs identical to consensus sequences from genotypes A, D and F, were selected for plasmid construction.
A total of eight recombinant plasmids designated as $\mathrm{M}$ Acons, M-A, S-A, M-Dcons, M-D, S-D, M-Fcons, and SFcons, were constructed. Among plasmids coding the HBV surface $\mathbf{M}$ protein, plasmids $\mathrm{M}$-Acons, $\mathrm{M}$-Dcons and $\mathrm{M}$ Fcons carry consensus sequences of genotypes A, D and F, respectively. Plasmids $\mathrm{M}-\mathrm{A}$ and $\mathrm{M}-\mathrm{D}$ encode variant $\mathrm{M}$ sequences of genotypes $\mathrm{A}$ and $\mathrm{D}$ that were also detected after sequencing of the clones. Plasmids that carry $\mathrm{S}$ protein sequences are S-A (variant S protein of genotype A), S-D (variant $S$ protein, genotype $\mathrm{D}$ ) and $\mathrm{S}$-Fcons (consensus $\mathrm{S}$ protein, genotype F). Plasmids M-Acons, M-A and S-A, as well as, M-Dcons, M-D and S-D, have identical S regions, with the exception of the amino acid substitutions T143M, found in plasmids M-A and S-A, and T125M, in plasmids M$\mathrm{D}$ and $\mathrm{S}-\mathrm{D}$. The comparison of $\mathrm{M}$ and $\mathrm{S}$ amino acid sequences deduced from recombinant plasmids, with a consensus sequence from genotype A, is shown in Figure 1. Additionally, two consensus sequences, one representing genotype $\mathrm{D}$ and other representing genotype $\mathrm{F}$, were added into the alignment for identification of genotype linked substitutions. Most of the amino acid variations occurring in HBV sequences of the expression plasmids were genotype-specific replacements. However, amino acid substitutions T125M and T143M, both located within the $a$ determinant of HBsAg, were specific variations of each HBV isolate (Figure 1).

\section{HBsAg Detection by ELISA}

To avoid a possible impact on transfection efficiency due to differences in plasmid quality, four independent transfection assays were performed using different plasmid preparations of each construct. Figure 2 shows the levels of HBsAg detected in culture medium of $\mathrm{CHO}$ cells after transient transfection assays with the recombinant plasmids. Among plasmids coding the consensus sequences of $\mathbf{M}$ protein, plasmid $\mathbf{M}$ Acons displayed the highest values of HBsAg in all transfection assays in comparison with genotypes $\mathrm{D}$ and $\mathrm{F}$ (Figure $2 \mathrm{a}$ ). The mean values showed a reduction of $30 \%$ and $37 \%$ in HBsAg detection values for genotypes $\mathrm{F}$ and $\mathrm{D}$, respectively, in comparison with genotype A (Figure 2a). Median values were very similar to mean values, indicating that results did not vary considerably from one experiment to another (Figure 2a).

Interestingly, the presence of the single amino acid variation T125M in plasmid M-D conferred an increase of 34\% in mean values of HBsAg in comparison with plasmid M-Dcons. In addition, plasmid M-A that had the unique substitution T143M, displayed a reduction of $44 \%$ in mean values of HBsAg in comparison with plasmid M-Acons (Figure 2b).

The comparison between $\mathrm{M}$ and S surface proteins, showed higher HBsAg detection levels for $\mathrm{M}$ constructs than their respective $\mathrm{S}$ plasmids. The mean values of HBsAg detection observed for M-A, M-D and M-Fcons plasmids were 5\%, 22\% and $43 \%$ higher than their $\mathrm{S}$ forms, respectively (Figure 2c).

Although values of HBsAg detected in cell extracts were lower than in medium, all results obtained for extracellular 
Figure 1. Differences in amino acid sequence alignment of $\mathrm{M}$ and $\mathrm{S}$ regions between constructed plasmids and a consensus sequence of genotype A. Consensus sequence of genotype A was originated from an alignment of $100 \mathrm{HBV}$ complete genome sequences from genotype A available in GenBank. Two other consensus sequences, one of genotype D and other from genotype F, originated from 35 and 44 GenBank complete genome sequences, respectively, were used to identify substitutions linked to genotypes. Shaded amino acids indicate variations specific for each HBV isolate. Amino acid positions corresponding to $a$ determinant are underlined.

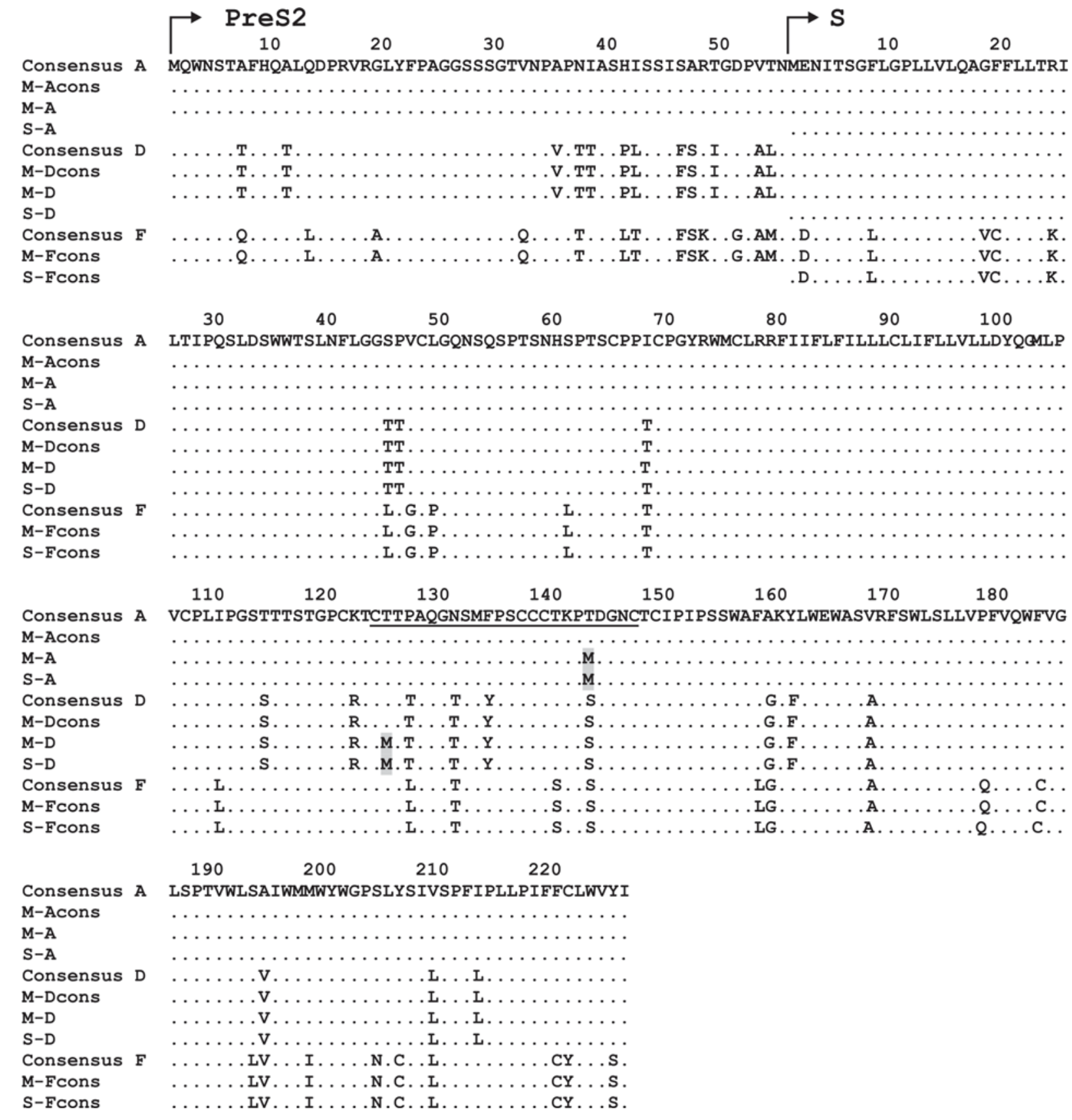

HBsAg were also observed for intracellular HBsAg (not shown).

\section{Discussion}

Due to the lack of proofreading activity of reverse transcriptase, HBV exhibits a mutation rate more than 10-fold higher than other DNA viruses. The evolutionary rate for the HBV genome in chronic hepatitis B is about $1.4 \times 10^{-5}$ to $3.2 \times 10^{-5}$ substitutions/site per year [20]. The different HBV genotypes are stable forms of the virus, which are the result of random changes selected over years of population pressure. On the other hand, HBV mutants arise in individuals under medically or naturally (chronic hepatitis B) induced immune pressure [18]. It is known that some specific mutations at the critical positions within HBsAg, most of them 
Figure 2. Extracellular HBsAg levels detected by ELISA after transfection of $\mathrm{CHO}$ cells with the constructed plasmids. HBsAg detection values are shown relative to M-Acons values. (a) - HBsAg values obtained in each transfection assay for $\mathrm{M}$ plasmids carrying consensus sequences, and the mean and median values from four independent experiments; (b) - HBsAg mean values of consensus and variant M plasmids; (c) - HBsAg mean values of M plasmids and their respective $\mathrm{S}$ constructs.

\section{A}

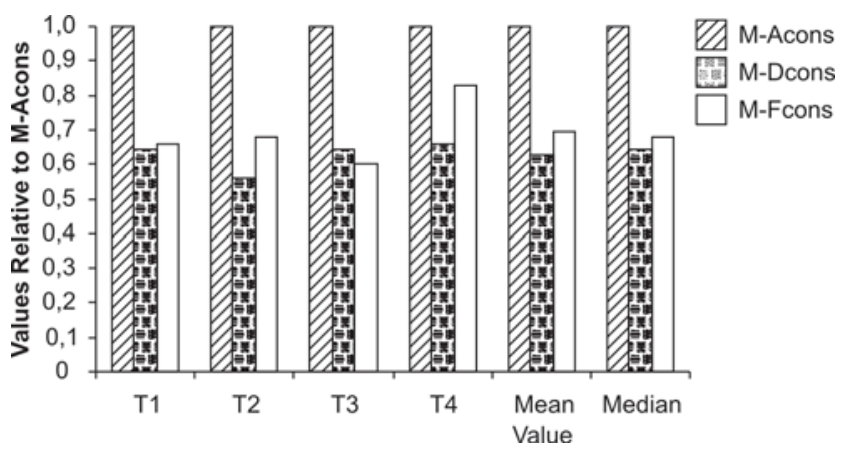

B

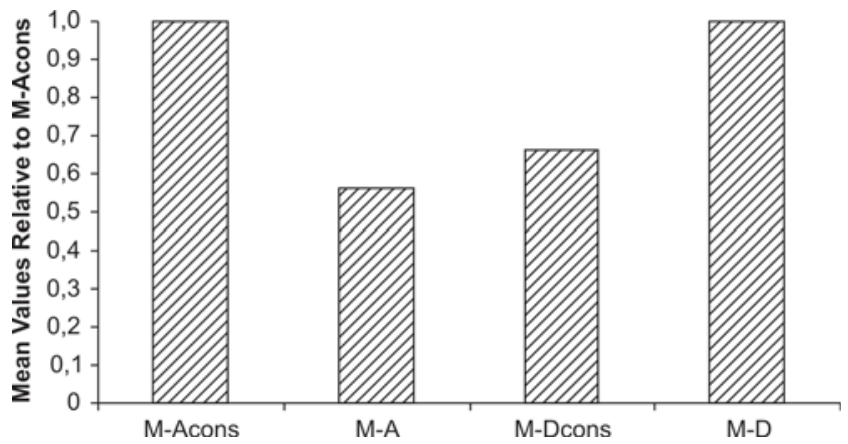

C

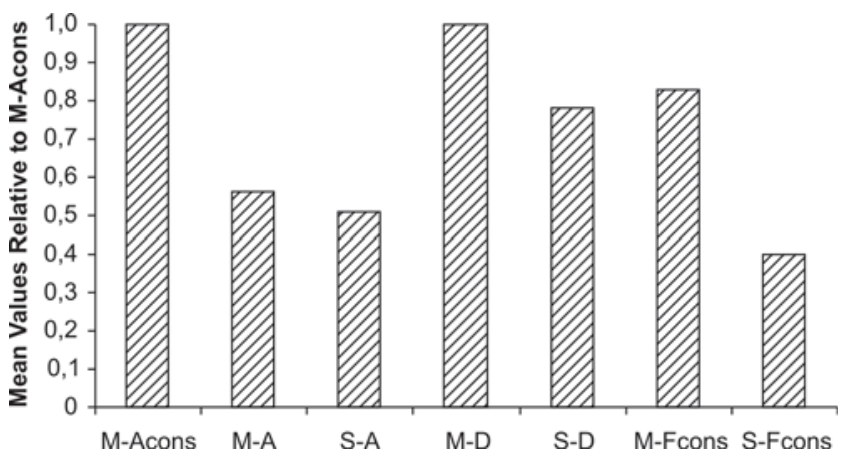

located in and around the $a$ determinant, may change the antigenicity of HBsAg [14, 21-24]. However, the influence of amino acid variations that are genotype-specific replacements of each genotype on the sensitivity of HBsAg commercial assays, is a matter that needs to be further investigated.
In the present study, plasmids containing genomic regions that express the two (M and S) surface proteins of HBV strains from the three (A, D and F) genotypes found in Brazil, were constructed and transfected into mammalian CHO cells. The extracellular and intracellular HBsAg levels produced by these constructs were evaluated by a commercial detection assay that uses polyclonal antibodies for both capture and detection. A previous work that analyzed the ability of seven commercial diagnostic assays, including the one used in our study, to detect HBsAg variants, demonstrated that the samples were better detected by polyclonal than monoclonal antibodies based assays [14]. Herein, detectable levels of HBsAg were observed in both medium and extracts of cells transfected with all constructed plasmids. However, the detection values of HBsAg varied among the different constructs.

Plasmids coding $\mathrm{M}$ protein from genotypes $\mathrm{A}, \mathrm{D}$ and $\mathrm{F}$ displayed higher levels of HBsAg detection than their respective $\mathrm{S}$ constructs. This may be explained by the presence of polyclonal antibodies in the commercial immunoassay used here. Therefore, $\mathrm{M}$ proteins were captured and detected by antibodies generated against both preS2 and $S$ regions, while $S$ proteins were only detected by anti-S antibodies.

The possible influence of HBV genotypes on HBsAg detection assays may be due to the presence in and around the $a$ determinant of several amino acid variations that are linked to each genotype. The set of amino acid variations T131N, S143T and G159A; T114S, K122R, P127T, F134Y and Y161F; and I110L, P127L, T140S and F158L are natural substitutions linked to genotypes A, D and F, respectively. Among the consensus forms of $M$ protein, the highest value of HBsAg, detected by the immunoassay used here, was for genotype A, followed by $\mathrm{F}$ (reduction of $30 \%$ in comparison with genotype A) and finally by D (reduction of $37 \%$ in comparison with genotype A). Recently, one study has suggested a correlation between HBV genotypes (A-D) and differential levels of extracellular HBsAg [25]. In this study, levels of secreted HBsAg were most abundant for genotype A and remotely for D, which was attributed to a higher transcription efficiency of preS/ $\mathrm{S}$ mRNA by genotype A than genotype D. However, in this case, HBsAg expression was performed by plasmids carrying the entire $\mathrm{HBV}$ genome, and thus, the $\mathrm{HBV}$ natural promoters were used in transcription process. Herein, HBsAg expression was under the strong CMV promoter and it is expected the same efficiency of transcription for all constructs. In our study, the sets of amino acid variations linked to genotypes $\mathrm{D}$ and $\mathrm{F}$ may have influenced HBsAg detection levels, at least by the commercial assay used here. In fact, the sensitivity of four commercial immunoassays for the detection of HBsAg from genotypes $\mathrm{A}$ to $\mathrm{F}$ has been previously tested. The results showed that in the concentration range between 0.1 and 0.5 $\mathrm{ng} / \mathrm{mL}$, one test failed to detect genotypes A to D and F, and other test failed to detect genotype F [18]. 
Interestingly, the presence of the unique amino acid variations T125M in plasmid M-D and T143M in plasmid M-A changed considerably the detection values of HBsAg in comparison with their consensus plasmids. These results indicate that a single variation may have more influence on HBsAg detection levels than the set of amino acids characteristic of each HBV genotype. T143M variation, that promoted a reduction of $44 \%$ on HBsAg mean values of $M$ protein from genotype $\mathrm{A}$, has been previously detected in vaccinated and non-vaccinated children [26] and in three asymptomatic individuals (one HBsAg negative, vaccinated person, and two HBsAg positive, non-vaccinated subjects) [27]. In the latter study, T143M substitution was detected along with the vaccine escape mutation G145R. Furthermore, it has been reported failure of some commercial assays to detect mutants with an amino acid substitution at position $143[28,29]$.

The other amino acid variation within the $a$ determinant of HBsAg observed here, was T125M. From a total of 91 HBV complete genome sequences from genotype $\mathrm{D}$ available in GenBank, 16 (17,6\%) sequences exhibited a Met residue at position 125, whereas the remaining 75 (82,4\%) sequences had a Thr residue at this position. This variation represents a nonconservative amino acid substitution and therefore, it may influence the conformation of HBsAg $a$ determinant and the binding of HBsAg-specific antibodies. A previous study has shown that the presence of the single T125M substitution within the HBsAg did not inhibit the binding with polyclonal and monoclonal antibodies [30]. In our study, T125M substitution led to an increase of $34 \%$ on HBsAg mean values of $\mathrm{M}$ protein from genotype $\mathrm{D}$ in comparison with its consensus form. Therefore, the presence of T125M substitution may influence HBsAg conformation, leading to an improvement on HBsAg detection levels by the commercial assay used here. Further studies should be conducted using plasmids carrying the complete HBV genome, as well as, different expression systems and commercial assays to analyze the influence of these substitutions on HBsAg detection levels.

In conclusion, the detection by a commercial immunoassay of HBsAg produced by recombinant plasmids, carrying the coding regions for HBV surface proteins $\mathrm{M}$ and $\mathrm{S}$ from genotypes A, D and F, showed an influence of HBV genotypes on HBsAg detection levels. However, unique amino acid substitutions not linked to genotypes, such as T125M and T143M described here, should have more implications in HBV immunological diagnostics than the natural amino acid variations characteristic of each HBV genotype.

\section{Acknowledgements}

We thank the Genomic Platform-DNA Sequencing PDTIS/ FIOCRUZ. This work was supported by Conselho Nacional de Desenvolvimento Científico e Tecnológico (CNPq) and by Fundação Carlos Chagas Filho de Amparo à Pesquisa do Estado do Rio de Janeiro (FAPERJ).

\section{References}

1. Arauz-Ruiz P, Norder H, Robertson BH, Magnius LO. Genotype $\mathrm{H}$ : a new Ameridian genotype of hepatitis $\mathrm{B}$ virus revealed in Central America. J Gen Virol 2002; 83:2059-2073.

2. Norder H, Hammas B, Lofdahl S, et al. Comparison of the amino acid sequences of nine different serotypes of hepatitis B surface antigen and genomic classification of the corresponding hepatitis B virus strains. J Gen Virol 1992; 73:1201-1208.

3. Stuyver L, De Gendt S, Van Geyt C, et al. A new genotype of hepatitis B virus: complete genome and phylogenetic relatedness. J Gen Virol 2000; 81:67-74.

4. Norder H, Hammas B, Lee SD, et al. Genetic relatedness of hepatitis $B$ viral strains of diverse geographical origin and natural variations in the primary structure of the surface antigen. J Gen Virol 1993; 74:1341-1348.

5. Norder H, Courouce AM, Coursaget P, et al. Genetic diversity of hepatitis B virus strains derived worldwide: genotypes, subgenotypes, and HBsAg subtypes. Intervirology 2004; 47:289309.

6. Araujo NM, Mello FC, Yoshida CF, et al. High proportion of subgroup A' (genotype A) among Brazilian isolates of Hepatitis B virus. Arch Virol 2004; 149:1383-1395.

7. de Castro L, Araujo NM, Sabino RR, et al. Nosocomial spread of hepatitis B virus in two hemodialysis units, investigated by restriction fragment length polymorphism analysis. Eur J Clin Microbiol Infect Dis 2000; 19:531-537.

8. Mello FC, Souto FJ, Nabuco LC, et al. Hepatitis B virus genotypes circulating in Brazil: molecular characterization of genotype $\mathrm{F}$ isolates. BMC Microbiol 2007; 7:103.

9. Bruss V, Ganem D. The role of envelope proteins in hepatitis B virus assembly. Proc Natl Acad Sci USA 1991; 88:1059-1063.

10. Huovila AP, Eder AM, Fuller SD. Hepatitis B surface antigen assembles in a post-ER, pre-Golgi compartment. J Cell Biol 1992; 118:1305-1320.

11. Ueda K, Tsurimoto T, Matsubara K. Three envelope proteins of hepatitis B virus: large S, middle S, and major S proteins needed for the formation of Dane particles. J Virol 1991; 65:35213529.

12. Magnius LO, Norder H. Subtypes, genotypes and molecular epidemiology of the hepatitis B virus as reflected by sequence variability of the S-gene. Intervirology 1995; 38:24-34.

13. Cooreman MP, Leroux-Roels G, Paulij WP. Vaccine- and hepatitis B immune globulin-induced escape mutations of hepatitis B virus surface antigen. J Biomed Sci 2001; 8:237-247.

14. Ireland JH, O'Donnell B, Basuni AA, et al. Reactivity of 13 in vitro expressed hepatitis B surface antigen variants in 7 commercial diagnostic assays. Hepatology 2000; 31:1176-1182.

15. Ly TD, Servant-Delmas A, Bagot $S$, et al. Sensitivities of four new commercial hepatitis B virus surface antigen (HBsAg) assays in detection of HBsAg mutant forms. J Clin Microbiol 2006; 44:2321-2326.

16. Seddigh-Tonekaboni S, Waters JA, Jeffers S, et al. Effect of variation in the common "a" determinant on the antigenicity of hepatitis B surface antigen. J Med Virol 2000; 60:113-121.

17. Schaefer S. Hepatitis B virus: significance of genotypes. J Viral Hepat 2005; 12:111-124.

18. Weber B. Genetic variability of the $\mathrm{S}$ gene of hepatitis B virus: clinical and diagnostic impact. J Clin Virol 2005; 32:102-112.

19. Niel C, Moraes MTB, Gaspar AMC, et al. Genetic diversity of hepatitis B virus strains isolated in Rio de Janeiro, Brazil. J Med Virol 1994; 44:180-186.

20. Okamoto H, Imai M, Kametani M, et al. Genomic heterogeneity of hepatitis B virus in a 54-year-old woman who contracted the infection through materno-fetal transmission. Jpn J Exp Med 1987; 57:231-236.

21. Coleman PF, Chen YC, Mushahwar IK. Immunoassay detection of hepatitis B surface antigen mutants. J Med Virol 1999; 59:1924. 
22. Cooreman MP, van Roosmalen MH, te Morsche R, et al. Characterization of the reactivity pattern of murine monoclonal antibodies against wild-type hepatitis B surface antigen to G145R and other naturally occurring "a” loop escape mutations. Hepatology 1999; 30:1287-1292.

23. Jolivet-Reynaud C, Lesenechal M, O’Donnell B, et al. Localization of hepatitis B surface antigen epitopes present on variants and specifically recognised by anti-hepatitis B surface antigen monoclonal antibodies. J Med Virol 2001; 65:241-249.

24. Zuckerman AJ, Zuckerman JN. Molecular epidemiology of hepatitis B virus mutants. J Med Virol 1999; 58:193-195.

25. Sugiyama M, Tanaka Y, Kato T, et al. Influence of hepatitis B virus genotypes on the intra- and extracellular expression of viral DNA and antigens. Hepatology 2006; 44:915-924.
26. Hsu HY, Chang MH, Liaw SH, et al. Changes of hepatitis B surface antigen variants in carrier children before and after universal vaccination in Taiwan. Hepatology 1999; 30:1312-1317.

27. Thakur V, Kazim SN, Guptan RC, et al. Transmission of G145R mutant of HBV to an unrelated contact. J Med Virol 2005; 76:40-46.

28. La'ulu SL, Roberts WL. The analytic sensitivity and mutant detection capability of six hepatitis B surface antigen assays. Am J Clin Pathol 2006; 125:748-751.

29. Levicnik-Stezinar S. Hepatitis B surface antigen escape mutant in a first time blood donor potentially missed by a routine screening assay. Clin Lab 2004; 50:49-51.

30. Zheng X, Weinberger KM, Gehrke R, et al. Mutant hepatitis B virus surface antigens (HBsAg) are immunogenic but may have a changed specificity. Virology 2004; 329:454-464. 